\title{
TOWERS, LADDERS AND THE B. B. NEWMAN SPELLING THEOREM
}

\author{
G. CHRISTOPHER HRUSKA and DANIEL T. WISE
}

(Received 24 May 2000; revised 3 January 2001)

Communicated by S. Gadde

\begin{abstract}
The Spelling Theorem of B. B. Newman states that for a one-relator group $\left\langle a_{1}, \ldots \mid W^{n}\right\rangle$, any nontrivial word which represents the identity must contain a (cyclic) subword of $W^{ \pm n}$ longer than $W^{n-1}$. We provide a new proof of the Spelling Theorem using towers of 2-complexes. We also give a geometric classification of reduced disc diagrams in one-relator groups with torsion. Either the disc diagram has three 2-cells which lie almost entirely along the boundary, or the disc diagram looks like a ladder. We use this ladder theorem to prove that a large class of one-relator groups with torsion are locally quasiconvex.
\end{abstract}

2000 Mathematics subject classification: primary 20F06, 57M07, $20 \mathrm{~F} 67$.

Keywords and phrases: One-relator groups, towers, staggered 2-complexes, local quasiconvexity.

\section{Introduction}

As explained by Howie in [3], some of the main results about one-relator groups can be proven using towers, a method first made explicit in the theory of 3-manifolds, in Papakyriakopolous's proof of Dehn's Lemma [12]. In fact, the tower method is implicit in Magnus's original solution of the word-problem and Freiheitssatz for one-relator groups, $[7,8]$. The advantage of the tower method over the other methods of proof is that the arguments are more geometric and conceptual, rather than combinatorial.

The first main result of this paper is a tower proof in Section 4 of the following theorem (see [6, IV.5.5]).

THEOREM 1.1 (B. B. Newman Spelling Theorem). Let $U$ be a freely reduced word which represents the identity in $\left\langle a_{1}, \ldots, a_{r} \mid W^{n}\right\rangle$, where $W$ is freely and cyclically

The second author was partially supported by a grant from the NSF.

(C) 2001 Australian Mathematical Society 0263-6115/2001 \$A2.00+0.00 
reduced. Then $U$ contains a subword $V$ such that $V$ is a subword of the cyclic word $W^{ \pm n}$ and $|V|>\left|W^{n-1}\right|$.

The statement of the B. B. Newman spelling theorem was first announced in [11]. We refer to [4] for a substantial generalization as well as for references to various other proofs of the theorem given by Gurevich, and others.

As usual for theorems about certain one-relator groups, the same proof works for the fundamental groups of certain staggered 2-complexes-in this case the staggered 2-complexes whose 2-cells are attached by proper powers. Theorem 1.1 is a special case of Theorem 4.6 which treats the general staggered case.

The second main result of the paper is Theorem 5.2 which provides a geometric classification of reduced disc diagrams in one-relator groups with torsion. Roughly speaking, we show that either the disc diagram has three 2-cells which lie almost entirely along the boundary, or the disc diagram looks like a ladder (see Figure 3 ). We use this to give the following application:

THEOREM 1.2. Let $G=\left\langle a_{1}, \ldots, a_{r} \mid W^{n}\right\rangle$, where $n \geq|W|$. Then $G$ is locally quasiconvex.

In Section 6, we provide a tower proof of a Freiheitssatz for the fundamental groups of staggered 2-complexes. We also provide a tower proof of the combinatorial reducibility of staggered 2 -complexes.

\section{Towers and staggered 2-complexes}

A map $X \rightarrow Y$ between $C W$-complexes is said to be combinatorial provided that its restriction to each cell of $X$ is a homeomorphism onto a cell of $Y$. (Throughout this paper, the term cell will always mean open cell.) A $C W$-complex is combinatorial provided that the attaching map of each of its cells is a combinatorial map (after a suitable subdivision). All the spaces in this paper will be 2-dimensional combinatorial complexes, and all the maps between spaces will be combinatorial.

We now collect some background on towers which is due to Howie [2].

DEFinition 2.1 (Tower). A map $A \rightarrow B$ of connected $C W$-complexes is a tower provided that it can be expressed as a composition

$$
A=B_{n} \hookrightarrow \hat{B}_{n-1} \rightarrow B_{n-1} \hookrightarrow \cdots \hookrightarrow \hat{B}_{2} \rightarrow B_{2} \hookrightarrow \hat{B}_{1} \rightarrow B_{1} \hookrightarrow B,
$$

where the maps are alternately inclusions of subcomplexes and infinite cyclic coverings. In other words, the covers are regular and connected with infinite cyclic covering transformation group. 
Let $f: C \rightarrow B$ be a map of connected $C W$-complexes. A map $f^{\prime}: C \rightarrow A$ is a tower lift of $f$ provided that there is tower $g: A \rightarrow B$ such that the following diagram commutes:

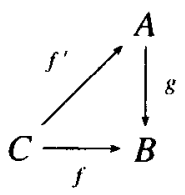

The tower lift $f^{\prime}$ is maximal if, for any tower lift $C \rightarrow D$ of $f^{\prime}$, the map $D \rightarrow A$ is an isomorphism.

LEMMA 2.2 ([2, Lemma 3.1]). Let $S$ be a compact $C W$-complex and $f: S \rightarrow K$ be a combinatorial map. Then $f$ has a maximal tower lifting.

DEFINITION 2.3 (Staggered). Let $K$ be a 2-complex such that the attaching map of each 2-cell is locally injective. We say that $K$ is staggered if there are linear orderings on the 2-cells and a subset $O$ of the 1-cells (called the ordered 1-cells) such that

(1) for each 2-cell $\alpha$, at least one ordered 1-cell is in the image of the attaching map of $\alpha$, and

(2) for 2-cells $\alpha$ and $\beta$, if $\alpha<\beta$ then $(\min \alpha)<(\min \beta)$ and $(\max \alpha)<(\max \beta)$, where $(\min \alpha)$ and $(\max \alpha)$ are respectively the least and greatest ordered 1-cells in the attaching map of $\alpha$.

A group presentation is staggered if its standard 2-complex is staggered.

The notion of staggered defined above appears in [6, page 152] and was implicit in Magnus' original proof of the Freiheitssatz [7]. Howie used a slightly less general definition in [3] to prove Lemma 2.6 and Lemma 2.4 below. His proofs work for the more general version of staggered that we use here, but we provide the details for the benefit of the reader.

LEMMA 2.4 ([3, Lemma 2]). If $Y \rightarrow X$ is a tower, and $X$ is a staggered 2-complex, then $Y$ is staggered.

PROOF. It is clear that a subcomplex of a staggered 2-complex is also staggered. Let $\phi: \hat{S} \rightarrow S$ be an infinite cyclic cover of a staggered 2-complex. We will show that $\hat{S}$ is staggered. Let $\gamma$ be a generator of the covering transformation group. Let the set $O$ of ordered 1-cells be the cells which project to ordered 1-cells in $S$. Linearly order $O$ and the 2-cells of $\hat{S}$ by the rule that $\alpha<\beta$ provided that either $\phi(\alpha)<\phi(\beta)$ or $\beta=\gamma^{n}(\alpha)$ for some $n \in \mathbb{Z}$ with $n>0$. Now let $\alpha<\beta$ be 2-cells of $\hat{S}$. If $\phi(\alpha)<\phi(\beta)$, then obviously $(\min \alpha)<(\min \beta)$. Otherwise $\beta=\gamma^{n}(\alpha)$ for some $n>0$, and so $(\min \beta)=\gamma^{n}(\min \alpha)$. An identical argument holds for $\max \alpha$. 
Convention 2.5. In general, if $X$ is staggered and $Y \rightarrow X$ is a tower, then $Y$ may be staggered in several ways. However, the arguments of this paper will always employ the staggering of $Y$ given in the proof of Lemma 2.4.

LEMMA 2.6 (Howie's Collapsing Lemma). Let $Y$ be a finite staggered 2-complex with at least one 2-cell and with $\mathrm{H}^{1}(Y)=0$. If the greatest 2-cell $\alpha$ of $Y$ is not attached along a proper power in $\pi_{1}\left(Y^{(1)}\right)$, then $Y$ collapses across $\alpha$ with free edge $\max \alpha$.

The following proof is identical to Howie's original proof which appeared as [3, Lemma 3].

PROOF. Note that, if some 2-cell $\gamma$ of $Y$ is attached along a proper power $s^{m}$ say, then replacing $\gamma$ by a 2-cell attached along $s$ will not change $H^{1}(Y)$. Nor will this procedure affect the staggering of $Y$.

We argue by induction on the number of 2-cells in $Y$, which by hypothesis is at least one. If there is only one 2-cell, then the first Betti number of the 1-skeleton $Y^{(1)}$ is at most 1 , since $\mathrm{H}^{1}(Y)=0$. On the other hand, since the attaching map $P$ of the 2-cell is a cyclically reduced path of positive length, and not a proper power, it follows that $Y^{(1)}$ cannot be a tree, and $P$ is the unique simple closed path in $Y^{(1)}$, whence the result.

For the inductive step, consider the Mayer-Vietoris sequence

$$
\cdots \longrightarrow \mathrm{H}^{1}(Y) \longrightarrow \mathrm{H}^{1}(Y-\alpha) \oplus \mathrm{H}^{1}\left(D^{2}\right) \longrightarrow \mathrm{H}^{1}\left(S^{1}\right) \longrightarrow \cdots
$$

associated to the adjunction of $\alpha$. From this we see that $\mathrm{H}^{1}(Y-\alpha)$ is at most cyclic. If the subcomplex $Y^{\prime}=Y-\{\alpha, \max \alpha\}$ is connected, then $\mathrm{H}^{1}(Y-\alpha)=\mathrm{H}^{1}\left(Y^{\prime}\right) \oplus \mathbb{Z}$, so $\mathrm{H}^{1}\left(Y^{\prime}\right)=0$. Otherwise, $Y^{\prime}$ has two components $Y_{1}$ and $Y_{2}$ say, and $\mathrm{H}^{1}(Y-\alpha)=$ $\mathrm{H}^{1}\left(Y_{1}\right) \oplus \mathrm{H}^{1}\left(Y_{2}\right)$. Without loss of generality we may assume $\mathrm{H}\left(Y_{1}\right)=0$. Also, in this case $Y_{1}$ cannot be a tree, since $\alpha$ is attached by a cyclically reduced closed path which meets $Y_{1}$.

Now apply the inductive hypothesis either to $Y^{\prime}$ or to $Y_{1}$, but with the staggering opposite to that inherited from $Y$ (that is, the orderings of cells is opposite). Then the complex in question collapses across its least 2-cell $\beta$ say (in the original ordering), with free edge $\min \beta$. But $\alpha$ does not involve $\min \beta$ since $\beta<\alpha$, so $Y$ also collapses across $\beta$ with free edge $\min \beta$. Let $Z=Y-\{\beta, \min \beta\}$ be the result of this collapse.

Then the inductive hypothesis applies to $Z$, so $Z$ collapses across $\alpha$ with free edge $\max \alpha$. But $\beta$ does not involve $\max \alpha$ since $\beta<\alpha$. Hence $Y=Z \cup\{\beta, \min \beta\}$ also collapses across $\alpha$ with free edge $\max \alpha$.

LEMMA 2.7. Let $Y$ be a finite staggered 2-complex which has no infinite cyclic cover. Then the greatest 2-cell of $Y$ is attached along a path $U^{n}$, where $U$ is a closed 
path in $Y$ that passes through $\max \alpha$ exactly once. Furthermore, no other 2-cell is attached along $\max \alpha$.

PROOF. Form a new 2-complex $Y^{\prime}$ from $Y$ by replacing the greatest 2-cell $\alpha$, which is attached along a path $U^{n}$ (where $U$ is not periodic), by a 2-cell $\alpha^{\prime}$ which is attached along the word $U$. The obvious map $Y \rightarrow Y^{\prime}$ is a $\pi_{1}$-surjection, and so $\mathrm{H}^{\prime}\left(Y^{\prime}\right)=0$. Now by Lemma 2.6 , we see that $U$ passes through $\max \alpha^{\prime}$ exactly once, and so the attaching path $U^{n}$ of $\alpha$ passes through $\max \alpha$ exactly once as well.

We make one final observation.

REMARK 2.8. Let $\phi: Y \rightarrow X$ be a tower map. For any 2-cell $\alpha$ of $Y$, if $\phi(\alpha)$ is attached along a path $U^{n}$ then $\alpha$ is attached along a path $V^{n}$. This is true for arbitrary towers because it holds for subcomplexes and infinite cyclic covers.

\section{Background on disc diagrams and spherical diagrams}

In this section we briefly review some background on disc diagrams and spherical diagrams. A more detailed account of this material can be found in [6].

A disc diagram $D$ is a planar, simply connected 2-complex. Given a 2-complex $X$ we will often study a map $D \rightarrow X$ and refer to this map as the disc diagram. Let $P \rightarrow X$ be a closed path which factors as $P \rightarrow D \rightarrow X$. We say that the disc diagram $D \rightarrow X$ is a disc diagram for $P \rightarrow X$ provided that $P$ maps onto $\partial D$, and furthermore for each 1-cell $e$ of $\partial D$, the preimage of $e$ in $P$ must consist of one or two 1 -cells according as to whether or not $e$ lies on the boundary of a 2-cell of $D$. The path $P$ is said to be a boundary path for $D$.

If the words $U$ and $V$ represent the same element of $\langle A \mid R\rangle$, then by a disc diagram for $U=V$ we will mean a disc diagram mapping to the standard 2-complex of $\langle A \mid R\rangle$ for the path corresponding to the word $U^{-1} V$.

We will occasionally employ the similar notion of a spherical diagram $D \rightarrow X$ which is a combinatorial map from a 2 -sphere to $X$.

A pair of (not necessarily distinct) 2-cells $C_{1}, C_{2}$ in a diagram $D$ which meet along a 1-cell $e$ of $D$, is a cancelable pair provided that the boundary cycles of $C_{1}$ and $C_{2}$ beginning with $e$ (in the same direction) are sent to identical paths in $X$ after composing with $D \rightarrow X$.

The disc [spherical] diagram $D \rightarrow X$ is said to be reduced provided that it has no cancelable pairs. It is a theorem of van Kampen, that a reduced disc diagram exists for any null-homotopic closed path (See [6, V.2.1]).

A spur in a disc diagram is the union of a valence one 0 -cell together with the 1 -cell incident with it.

We conclude this section with an easy lemma whose proof we leave to the reader. 
LEMMA 3.1. Let $D \rightarrow Y$ be a tower lift of a reduced disc [spherical] diagram $D \rightarrow X$. Then $D \rightarrow Y$ is itself a reduced disc [spherical] diagram.

\section{Tower proof of the B. B. Newman Spelling Theorem}

In this section we prove Theorem 4.6 and Theorem 4.8 which are the first main results of the paper. The crucial geometric tool employed in the proof of these theorems is Lemma 4.5 which will also play a key role later in Section 5.

We begin the section by applying Lemma 2.7 to a maximal tower lift of a reduced disc [spherical] diagram. In this situation, the diagram has a useful geometric decomposition into smaller subdiagrams.

LEMMA 4.1 (On the Boundary). Let $D \rightarrow Y$ be a maximal tower lift of a reduced disc [spherical] diagram $D \rightarrow X$ with $X$ a staggered 2-complex. Let $A$ be a 2-cell of $D$ which maps to the greatest 2-cell $\alpha$ of $Y$. Let $n$ be the positive number such that $\alpha$ is attached along a path $U^{n}$, where $U$ is not homotopic to a periodic path in $Y^{(1)}$. Then $\partial A$ contains exactly $n 1$-cells $E_{1}, \ldots E_{n}$ which map to $\max \alpha$, and each $E_{i}$ lies in $\partial D$.

Note that in the spherical case, $\partial D$ is empty, and so the lemma asserts that no such reduced spherical diagram exists.

Proof. Observe that $Y$ has no infinite cyclic cover and $D \rightarrow Y$ is surjective, so $Y$ is finite. By Lemma 2.7 , the path $U$ passes through $\max \alpha$ exactly once. Furthermore, no other 2 -cell is attached along $\max \alpha$.

To see that each $E_{i}$ is contained in $\partial D$ note that if $E_{i}$ were an interior 1-cell, then it would appear in two different ways in the boundary of a 2-cell. Since each such 2-cell would map to $\alpha$, this would form a cancelable pair. This is impossible because $D \rightarrow X$ is reduced and therefore by Lemma 3.1 $D \rightarrow Y$ is reduced. It follows that each $E_{i}$ is contained in $\partial D$. Since $\partial A$ passes $n$ times through maximal 1-cells, and $\partial A$ passes through each exactly once, we see that there are $n$ distinct maximal 1 -cells on $\partial A$ as claimed.

Definition 4.2 (Branches). Let $A, D, Y$, and $\left\{E_{1}, \ldots, E_{n}\right\}$ be as in Lemma 4.1 then the subcomplex $D-\left\{A, E_{1}, \ldots, E_{n}\right\}$ consists of $n$ path components. These components are the branches at $A$ with respect to $D \rightarrow Y$. A branch is trivial if it is contained in $\partial A$ and nontrivial otherwise.

Figure 1 illustrates a disc diagram with a dark 2-cell $A$ and four 1-cells mapping to $e$. There are four branches at $A$ and exactly one of these branches is trivial. 


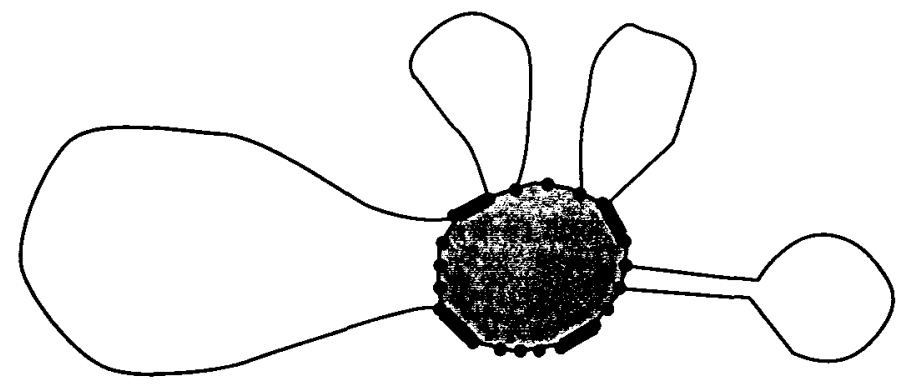

FIGURE 1.

Definition 4.3 ( $\lambda$-external). The 2-cell $R$ of $D$ is $\lambda$-external provided that there is a subpath $Q$ of the attaching path of $R$ such that:

(1) $Q$ is a subpath of the boundary path of $D$.

(2) $|Q|>\lambda|\partial R|$.

We will sometimes refer to $R$ as an external 2-cell when the value of $\lambda$ is clear from the context.

LEMMA 4.4. Let $D$ and $A$ be as in Lemma 4.1, let $P$ be a branch at $A$, and let $D^{\prime}$ be the subdiagram $P \cup \bar{A}$. Suppose $B \neq A$ is a 2-cell of $D^{\prime}$. If $B$ is $\lambda$-external for the diagram $D^{\prime}$, then $B$ is also $\lambda$-external for the diagram $D$.

Proof. By the definition of branch, the 2-cell $B$ does not share any 1-cells with any 2-cell of $D-D^{\prime}$. If a 1 -cell $E$ in $\partial B$ lies in $\partial D^{\prime}$, then it also lies in $\partial D$.

LEMMA 4.5 (Two external 2-cells). Let $X$ be a staggered 2-complex and let $D \rightarrow X$ be a reduced disc diagram. Suppose that $D$ has no spurs, and $D$ contains at least two 2-cells. Then D contains two distinct 2-cells $C_{1}$ and $C_{2}$ such that

(1) $C_{i}$ maps to a 2-cell $\gamma_{i}$ in $X$ whose attaching map is $U^{n_{i}}$, where $U$ is not homotopic to a periodic path in $X^{(1)}$;

(2) $C_{i}$ is an $\left(\left(n_{i}-1\right) / n_{i}\right)$-external 2-cell of $D$.

PROOF. We prove the statement by induction on the number of 2-cells in $D$. Lift $D \rightarrow X$ to a maximal tower:

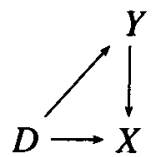

Suppose first that $Y$ has a unique 2-cell $\alpha$. Then every 2-cell of $D$ maps to $\alpha$, which is trivially the greatest 2-cell of $Y$. Let $A_{1}$ and $A_{2}$ be any two distinct 2-cells 
of $D$. If they each have only one nontrivial branch, then they are external 2-cells of $D$ and we are done. On the other hand, if one of these 2-cells (say $A_{1}$ ) has two nontrivial branches $B_{1}$ and $B_{2}$, then we can subdivide $D$ into two strictly smaller disc diagrams $D_{1}$ and $D_{2}$ with $D_{i}=B_{i} \cup \overline{A_{1}}$. By induction, each of these subdiagrams has two external 2-cells, at least one of which is distinct from $A$. Thus there are two distinct 2-cells (different from $A$ ) $C_{1} \subset D_{1}$ and $C_{2} \subset D_{2}$ that are each external for the corresponding subdiagram. But then Lemma 4.4 implies that $C_{1}$ and $C_{2}$ are each external 2-cells for the original diagram $D$ as well.

Now suppose that $Y$ has more than one 2-cell. Let $\alpha$ and $\kappa$ be respectively the greatest and least 2-cells of $Y$. Choose 2-cells $A$ and $K$ in $D$ mapping to $\alpha$ and $\kappa$. We can define branches of $K$ by reversing the ordering of the cells of $Y$. We now proceed as above, noting that $A$ and $K$ are distinct 2-cells. As before, if each of $A$ and $K$ has only one nontrivial branch, then we are done. Otherwise one of them splits the diagram into two strictly smaller subdiagrams, and the result follows from the inductive hypothesis.

At this point, we can easily prove the following form of the B. B. Newman Spelling Theorem.

THEOREM 4.6. Let $G=\left\langle x_{1}, \ldots \mid R_{1}^{n_{1}}, \ldots\right\rangle$ be a staggered presentation. Let $U$ be a freely and cyclically reduced word representing the identity of $G$. Then $U$ contains a subword $S$ such that $S$ is a subword of the cyclic word $R_{j}^{ \pm n_{j}}$ for some $j$ and $|S|>\left|R_{j}^{n_{j}-1}\right|$.

Proof. Let $X$ be the standard 2-complex for the presentation, and let $D \rightarrow X$ be a reduced disc diagram for $U$. It will be convenient to think of 1-cells of $X$ as being oriented and labeled by their corresponding generators. This pulls back to an orientation and label for each 1-cell of $D$. Thus a path in $D$ corresponds to a certain word in the generators and their inverses. To prove the theorem, we will find a certain 2-cell $C$ of $D$ mapping to an $R_{j}^{n_{j}}$ 2-cell of $X$. The boundary of $C$ will have a subpath $S$ corresponding to a subword of $U$ satisfying $|S|>\left|R_{j}^{n_{j}-1}\right|$.

Either $D$ has only one 2-cell $C$, in which case the result follows immediately, or we may apply Lemma 4.5 to $D$ and conclude that $D$ has two external 2-cells, $C_{1}$ and $C_{2}$. Notice that the path $U$ may begin at a point in the interior of at most one of these external 2-cells, say $C_{1}$. Then $C_{2}$ maps to an $R_{j}^{n_{j}} 2$-cell of $X$, and the external path of $C_{2}$ corresponds to a subword $S$ of $U$ with $|S|>\left|R_{j}^{n_{j}-1}\right|$ as required.

DEFINITION 4.7 (Intervals). Let $G=\left\langle x_{1}, \ldots \mid R_{1}^{n_{1}}, \ldots\right\rangle$ be a staggered presentation, and let $W$ be a freely reduced word. The interval spanned by $W$, denoted $I_{W}$, is the convex closure of the set of ordered letters of $W$ in the set of all ordered letters. In other words, $I_{W}$ is the smallest interval containing all the ordered generators in $W$. 


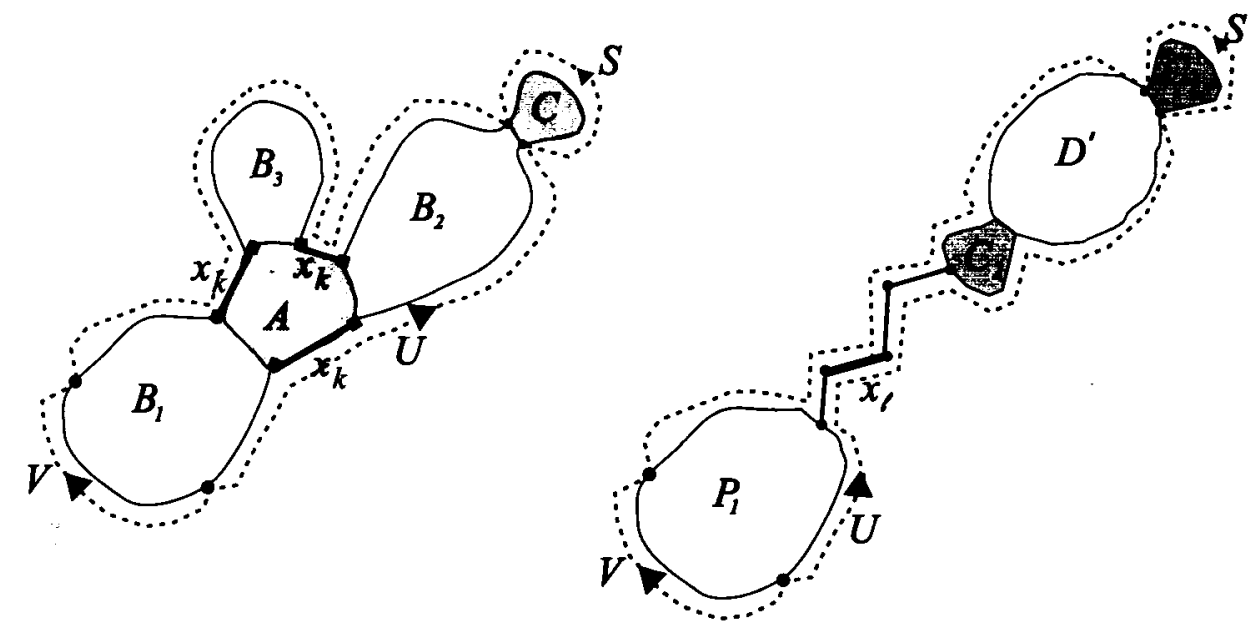

FIGURE 2. The diagram on the left corresponds to the case where $x_{k}$ is the greatest 1 -cell in $X$. The diagram on the right corresponds to the case where there is a greater 1-cell $x_{\ell}$ which is necessarily not on the boundary of any 2-cell. In both diagrams, the paths $U$ and $V$ begin and end at the bold 0 -cells. Note that the path $U$ wanders around quite a bit and contains the subpath $S$. Also note that on the left, $B_{2}$ is actually the union of the regions labeled by $B_{2}$ and $C$ in the figure, while on the right, $D^{\prime}$ is actually the union of the regions labeled $D^{\prime}, C_{1}$, and $C_{2}$ in the figure.

The following generalization of the B. B. Newman Spelling Theorem was first proved in [4].

THEOREM 4.8. Let $G=\left\langle x_{1}, \ldots \mid R_{1}^{n_{1}}, \ldots\right\rangle$ be a staggered presentation. Let $U$ and $V$ be two freely reduced words which represent the same element of $G$. Suppose that $I_{V}$ omits a generator $x_{i}$ which occurs in $I_{U}$. Then $U$ contains a subword $S$ such that $S$ is a subword of the cyclic word $R_{j}^{ \pm n_{j}}$ for some $j$ and $|S|>\left|R_{j}^{n_{j}-1}\right|$.

Proof. Let $X$ be the standard 2-complex for the presentation, and let $D \rightarrow X$ be a reduced disc diagram for $U={ }_{G} V$. As in Theorem 4.6, we will find a certain 2-cell $C$ of $D$ mapping to an $R_{j}^{n_{j}}$ 2-cell of $X$. The boundary of $C$ will have a subpath $S$ corresponding to a subword of $U^{-1}$ in the boundary path $U^{-1} V$ of $D$, and furthermore, $|S|>\left|R_{j}^{n_{j}-1}\right|$.

Notice that we cannot directly apply Lemma 4.5 since $D$ may have spurs, and we could not easily conclude that either of the long boundary paths produced by the lemma corresponds to a subword of $U^{-1}$. To proceed, we must find an appropriate subdiagram $D^{\prime}$ to which we apply Lemma 4.5.

Without loss of generality, we may assume that the generator $x_{i}$ which is omitted from $I_{V}$ is greater than the generators in $I_{V}$, since otherwise we can reverse the order 
of the staggering. As before, we begin by lifting $D \rightarrow X$ to a maximal tower:

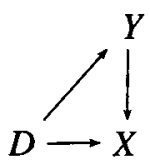

Let $\alpha$ be the greatest 2-cell of $Y$, and let $A$ be a 2-cell of $D$ which maps to $\alpha$. Suppose that $\alpha$ maps to the $R_{j}^{n_{j}} 2$-cell of $X$. Let $e$ be the greatest 1-cell in $\partial \alpha$ and let $x_{k}$ be the generator labeling the 1-cell it projects to in $X$. This generator $x_{k}$ must be the greatest generator occurring in the boundary of a 2-cell in the image of $D \rightarrow X$. Indeed, if there were a greater generator on the boundary of a 2-cell $R$ in the image $D \rightarrow X$, then as the staggering of $Y$ was defined in Lemma 2.4, $R$ would be greater than the image of $\alpha$, and thus a 2-cell $\beta$ of $Y$ which maps to $R$ would be greater than $\alpha$ which is a contradiction.

Suppose first that $x_{k}$ is the greatest generator in the image of $D \rightarrow X$. Then $\partial A$ contains 1-cells $E_{1}, \ldots, E_{n_{j}}$ which map to $x_{k}$ in $X$, and by Lemma 4.1, these 1-cells lie in $\partial D$. It follows that $x_{k}$ occurs in the word $U^{-1} V$, and since $x_{k} \geq x_{i}$ we see that $x_{k}$ must occur in $U$, but not in $V$. So the $V$ part of the boundary path $U^{-1} V$ of $D$ must lie inside one of the branches of $A$. Let $B_{1}$ be the branch of $A$ containing $V$. If $A$ has no nontrivial branches other than $B_{1}$, then $A$ is $\left(\left(n_{j}-1\right) / n_{j}\right)$-external for $D$. Otherwise, as illustrated on the left in Figure 2, $A$ has a nontrivial branch $B_{2} \neq B_{1}$. In this case, we can apply Lemma 4.5 to the subdiagram $D^{\prime}=\bar{A} \cup B_{2}$ and conclude that $D$ has an external 2-cell $C$ as above.

Now suppose that $x_{k}$ is not the greatest generator in the image of $D \rightarrow X$. Then the greatest generator $x_{\ell}$ appearing in the 1-skeleton of $D$ is not in the boundary of any 2-cell of $D$, and so is not in the interior of $D$. Thus, as illustrated on the right in Figure 2, $x_{\ell}$ appears in the boundary path $U^{-1} V$ of $D$. And again, since $x_{\ell} \geq x_{i}$ we see that $x_{\ell}$ must occur in $U$ but not in $V$. Consequently, some 1-cell of $D$ with label $x_{\ell}$ separates $D$ into two components $P_{1}$ and $P_{2}$. The $V$ subpath of the boundary path of $D$ lies entirely within one of these components (say $P_{1}$ ). Since $U$ is freely reduced, $P_{2}$ contains at least one 2-cell. More precisely, $P_{2}$ is the union of a spurless 2-complex $D^{\prime}$ and a (possibly trivial) chain of 1-cells starting at a vertex $v$ of $D^{\prime}$ and ending at a spur. (In the diagram on the right in Figure 2, $P_{2}$ is the union of $D^{\prime}$ and a chain of two 1-cells.) Applying Lemma 4.5 to $D^{\prime}$ provides 2-cells $C_{1}$ and $C_{2}$ which are external for $D^{\prime}$. Since $v$ can lie in the interior of at most one of the external paths of these 2-cells say $C_{2}$, we see that $C_{1}$ is the desired external 2-cell for $D$.

\section{Ladders}

The main result of this section is Theorem 5.2 which characterizes certain discdiagrams. An application is given in Theorem 5.7 to the local quasiconvexity of 

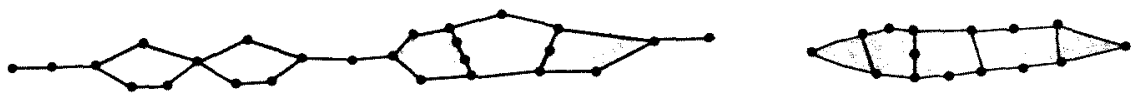

FIGURE 3.

certain one-relator groups with torsion. We begin with the following definition which is slightly different from the definition first introduced in [10] where ladders were studied in conjunction with small-cancellation theory.

DEFINITION 5.1 (Ladder). A disc diagram $D$ is a ladder provided that it is a union $\bigcup_{1 \leq i \leq n} \bar{C}_{i}$ where each $C_{i}$ is a 1 -cell or 2 -cell and:

(1) $\overline{C_{i}} \cap \overline{C_{j}}$ is nonempty if and only if $i$ and $j$ are consecutive.

(2) If $|i-j|=1$, then $\overline{C_{i}} \cap \overline{C_{j}}$ is a (possibly trivial) interval.

(3) If $|i-j|=1$ and $C_{i}$ is a 1-cell, then $\overline{C_{i}} \cap \overline{C_{j}}$ is a 0 -cell.

We refer the reader to Figure 3 for a picture of two ladders.

THEOREM 5.2 (Ladder). Let $X$ be a staggered 2-complex and suppose that there are constants $n$ and $k$ such that each 2-cell of $X$ is attached along a path $U^{n}$, where $U$ is a non-periodic path of length $k$.

Let $D \rightarrow X$ be a reduced disc diagram with no spurs. Then either:

(1) $D$ consists of a single 0 -cell.

(2) $D$ is a ladder with an $((n-1) / n)$-external 2 -cell at each end.

(3) $D$ has at least three $((n-2) / n)$-external 2-cells.

Proof. We induct on the number of 2-cells in $D$. A disc diagram $D$ with no spurs and a single 2-cell is a ladder. Now suppose $D$ has at least two 2-cells. As usual, we begin by lifting $D \rightarrow X$ to a maximal tower:

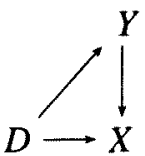

Let $\alpha$ be the maximal 2-cell of $Y$, and let $A$ be a 2-cell of $D$ which maps to $\alpha$. The argument splits into three cases corresponding to the number of branches at $A$.

Case $\geq 3$ : Suppose $A$ has at least three nontrivial branches $B_{1}, B_{2}$, and $B_{3}$. Let $D_{i}$ be the subdiagram $\bar{A} \cup B_{i}$. By Lemma 4.5 , each $D_{i}$ contains two $((n-1) / n)$-external 2-cells, at least one of which is distinct from $A$. So by Lemma $4.4, D$ has three 2-cells which are $((n-1) / n)$-external.

Case 2: Suppose $A$ has exactly two nontrivial branches $B_{1}$ and $B_{2}$. Then we have two strictly smaller diagrams $D_{1}=B_{1} \cup \bar{A}$ and $D_{2}=B_{2} \cup \bar{A}$. Notice that $A$ 

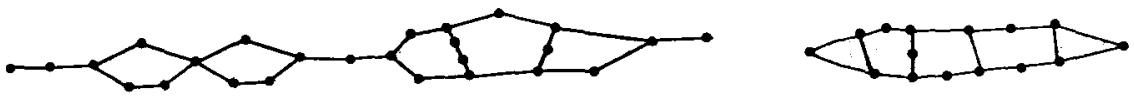

FIGURE 3.

certain one-relator groups with torsion. We begin with the following definition which is slightly different from the definition first introduced in [10] where ladders were studied in conjunction with small-cancellation theory.

DEFINITION 5.1 (Ladder). A disc diagram $D$ is a ladder provided that it is a union $\bigcup_{1 \leq i \leq n} \bar{C}_{i}$ where each $C_{i}$ is a 1-cell or 2-cell and:

(1) $\overline{C_{i}} \cap \overline{C_{j}}$ is nonempty if and only if $i$ and $j$ are consecutive.

(2) If $|i-j|=1$, then $\overline{C_{i}} \cap \overline{C_{j}}$ is a (possibly trivial) interval.

(3) If $|i-j|=1$ and $C_{i}$ is a 1-cell, then $\overline{C_{i}} \cap \overline{C_{j}}$ is a 0 -cell.

We refer the reader to Figure 3 for a picture of two ladders.

THEOREM 5.2 (Ladder). Let $X$ be a staggered 2-complex and suppose that there are constants $n$ and $k$ such that each 2-cell of $X$ is attached along a path $U^{n}$, where $U$ is a non-periodic path of length $k$.

Let $D \rightarrow X$ be a reduced disc diagram with no spurs. Then either:

(1) $D$ consists of a single 0 -cell.

(2) $D$ is a ladder with an $((n-1) / n)$-external 2 -cell at each end.

(3) $D$ has at least three $((n-2) / n)$-external 2-cells.

Proof. We induct on the number of 2-cells in $D$. A disc diagram $D$ with no spurs and a single 2-cell is a ladder. Now suppose $D$ has at least two 2-cells. As usual, we begin by lifting $D \rightarrow X$ to a maximal tower:

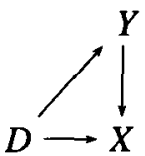

Let $\alpha$ be the maximal 2-cell of $Y$, and let $A$ be a 2-cell of $D$ which maps to $\alpha$. The argument splits into three cases corresponding to the number of branches at $A$.

Case $\geq 3$ : Suppose $A$ has at least three nontrivial branches $B_{1}, B_{2}$, and $B_{3}$. Let $D_{i}$ be the subdiagram $\bar{A} \cup B_{i}$. By Lemma 4.5 , each $D_{i}$ contains two $((n-1) / n)$-external 2-cells, at least one of which is distinct from $A$. So by Lemma $4.4, D$ has three 2-cells which are $((n-1) / n)$-external.

Case 2: Suppose $A$ has exactly two nontrivial branches $B_{1}$ and $B_{2}$. Then we have two strictly smaller diagrams $D_{1}=B_{1} \cup \bar{A}$ and $D_{2}=B_{2} \cup \bar{A}$. Notice that $A$ 
is $((n-1) / n)$-external for both $D_{1}$ and $D_{2}$. By Lemma 4.5 , each $D_{i}$ contains two $((n-1) / n)$-external 2-cells. If one of the $D_{i}$ (say $\left.D_{1}\right)$ has three $((n-2) / n)$-external 2-cells, then by Lemma 4.4 , at least two of these 2 -cells are $((n-2) / n)$-external for $D$. But $D$ also has an $((n-1) / n)$-external 2 -cell in $B_{2}$, so that $D$ has a total of at least three 2 -cells which are $((n-2) / n)$-external.

We can therefore assume that each of $D_{1}$ and $D_{2}$ has at most two $((n-2) / n)$ external 2-cells one of which must be $A$. Furthermore, since $D=D_{1} \cup_{\bar{A}} D_{2}$ and $D$ has no spurs, we see that neither $D_{1}$ nor $D_{2}$ has a spur. So $D_{1}$ and $D_{2}$ are each spurless reduced disc diagrams consisting of more than a single 0 -cell. Thus by induction, the only possibility is that they are each ladders. Furthermore we can assume that $A$ is the last cell of $D_{1}$ and that $A$ is the first cell of $D_{2}$ because if $A$ is not at the end then there would be three $((n-2) / n)$-external 2-cells.

It is easy to check that $D$ is a ladder because $D$ is the union of these two ladders along $A$, and thus $D$ can be expressed as the union of a sequence of 1-cells and 2-cells by concatenating the sequences for $D_{1}$ and $D_{2}$. The intersection conditions in the definition of a ladder follow because $\bar{B}_{1}$ and $\bar{B}_{2}$ are disjoint.

Case 1: Now suppose that $A$ has only one nontrivial branch $B$. Further suppose that $D$ does not satisfy possibility (3) of the theorem. Notice that $A$ is $((n-1) / n)$-external for $D$. Let $D^{\prime}$ be the subdiagram obtained from $B$ by repeatedly removing spurs until no spurs remain. Since $D$ has no spurs, $D^{\prime}$ must consist of more than a single 0 -cell. We will now show that $D^{\prime}$ does not satisfy possibility (3) of the theorem, so that $D^{\prime}$ must be a ladder by induction.

If $D^{\prime}$ contains more than one 2-cell, then by Lemma $4.5, D^{\prime}$ has two $((n-1) / n)$ external 2-cells $C_{1}$ and $C_{2}$. By the hypothesis that $D$ does not satisfy possibility (3), only one of these, say $C_{1}$, can be $((n-2) / n)$-external for $D$. Let $\gamma$ be a subpath of the boundary path of $C_{2}$ which is also a subpath of the boundary path of $D^{\prime}$ and which has more than $(n-1) k$ edges.

Define a subpath $\sigma$ of the boundary path of $D^{\prime}$ as follows. If $\partial A$ intersects $\partial D^{\prime}$, then let $\sigma$ be that intersection. Otherwise, $A$ is connected to $D^{\prime}$ by a chain of isolated 1 -cells. In this case, let $\sigma$ be the trivial path at the 0 -cell where this chain meets $D^{\prime}$. Notice that the length of $\sigma$ is less than $k$. Further, $\gamma$ cannot contain a subpath with more than $(n-2) k$ edges which is also a subpath of the boundary path of $D$, since otherwise $C_{2}$ would be $((n-2) / n)$-external for $D$. So $\sigma$ must lie in the interior of $\gamma$. Thus if $E$ is any 2-cell of $D^{\prime}$ other than $C_{2}$, then $E$ is $\lambda$-external for $D^{\prime}$ if and only if $E$ is $\lambda$-external for $D$. So $D^{\prime}$ is a spurless reduced disc diagram which fails to satisfy possibilities (1) and (3) of the theorem, and, hence, $D^{\prime}$ must be a ladder with $C_{1}$ and $C_{2}$ at the two ends. Attaching $A$ (and possibly an intermediate chain of isolated 1-cells) to $D^{\prime}$ along an interior subpath of $\gamma$, we see that $D$ is, in fact, also a ladder.

REMARK 5.3. A more careful argument would provide a stronger version of Theo- 


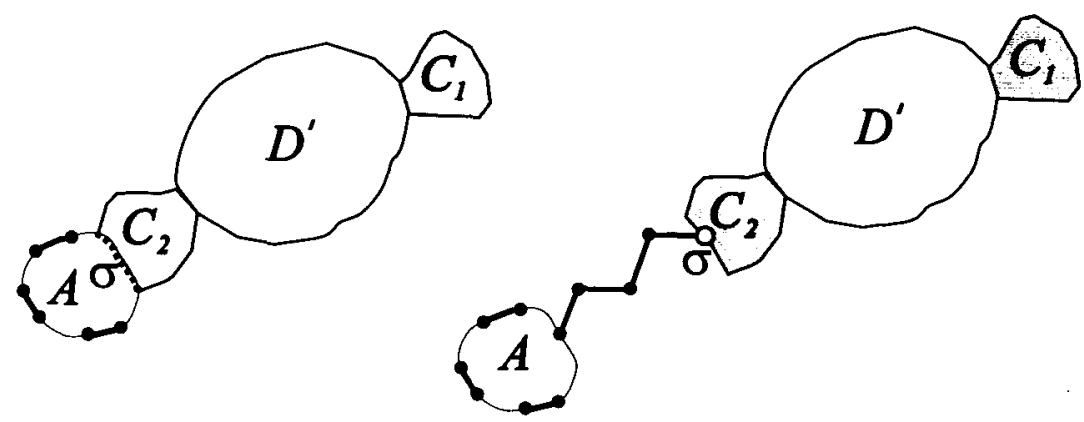

FIGURE 4. The diagram on the left corresponds to the case where $\partial A$ intersects $\partial D^{\prime}$. The diagram on the right is the case where $A$ is connected to $D^{\prime}$ by a chain of isolated 1-cells. Note that in each case $D^{\prime}$ is the union of the regions labeled by $D^{\prime}, C_{1}$, and $C_{2}$ in the diagram.

rem 5.2 where we replace the third possibility in the conclusion by

3. $D$ has at least three $\lambda$-external 2-cells, where $\lambda=((n-2) k+1) / n k$.

The following useful variation allowing spurs can be deduced immediately from Theorem 5.2.

THEOREM 5.4. Let $X$ be a staggered 2-complex and suppose that there are constants $n$ and $k$ such that each 2-cell of $X$ is attached along a path $U^{n}$, where $U$ is a nonperiodic path of length $k$.

Let $D \rightarrow X$ be a reduced disc diagram. Then either:

(1) D consists of a single 0 -cell.

(2) $D$ is a ladder with a spur or $((n-1) / n)$-external 2 -cell at each end.

(3) D has at least three spurs and/or $((n-2) / n)$-external 2-cells.

EXAMPLE 5.5. We now provide an example of a staggered 2-complex $X$ and a reduced disc diagram $D \rightarrow X$ such that $D$ is not a ladder, but $D$ does not have three $((n-1) / n)$-external 2-cells.

Let $X$ be the standard 2-complex of the one-relator presentation $\left\langle a, b \mid(a b a b a)^{n}\right\rangle$ and consider the word

$$
W=b a(a b a b a)^{n-1} a(a b a b a)^{n-1} a b(a b a b a)^{-(n-1)} .
$$

Then $W$ is the boundary path of a reduced disc diagram $D \rightarrow X$ with three 2-cells, and only two of these are $((n-1) / n)$-external, but $D$ is not a ladder. See Figure 5 for an illustration of this disc diagram in the case $n=3$.

In conjunction with a result from [9], Theorem 5.2 yields a powerful result about subgroups of certain one-relator groups with torsion. We refer the reader to [13] for 


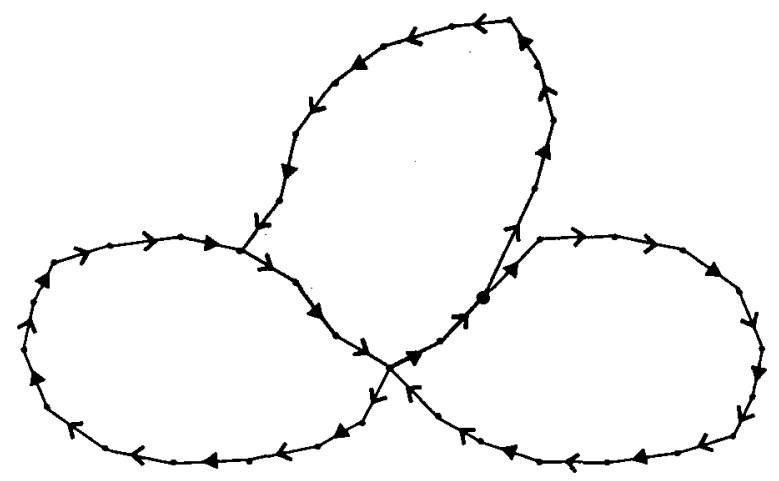

FIGURE 5.

the notion of a quasiconvex subgroup. We refer the reader to [9] for the notions of weighted 2-complex, perimeter, sides, and weight of a 2-cell.

We will apply the following result from [9].

THEOREM 5.6. A compact weighted 2-complex $X$ has a locally quasiconvex fundamental group provided that there exists $K$ such that the following condition holds:

Let $P \rightarrow \tilde{X}$ be any locally injective path and let $J \rightarrow \tilde{X}$ denote a geodesic with the same endpoints as $P$, and suppose that $|P|>K|J|$. Then there exists a 2-cell $R$ of $X$ attached along a path $W^{n}$ such that $W^{n}$ is a concatenation $Q S$ where $Q$ is $a$ subpath of $P \rightarrow X$ and Perimeter $(S)<n$ Weight $(R)$.

THEOREM 5.7. Let $G=\left\langle a_{1}, \ldots, a_{r} \mid W^{n}\right\rangle$. If $n \geq|W|$, then $G$ is locally quasiconvex.

ProOF. Without loss of generality we can assume that $W$ is cyclically reduced. We can assume that $n \geq 4$ because otherwise $G$ is virtually free and so the theorem is well-known [13]. Similarly, the theorem is true if $|W|=1$ and so we will assume that at least two distinct letters occur in $W$. We will now apply Theorem 5.6 for an appropriate weighting on the sides of the 2-cells of the standard 2-complex $X$ of the presentation for $G$. Let $K=n|W|$. Let $P$ denote a path in $\tilde{X}$ and let $J$ denote a geodesic with the same endpoints as $P$. Let $D$ denote a disc diagram for $J=P$. By Theorem 5.4, either $D$ has at least three spurs and/or $((n-2) / n)$-external 2 -cells or $D$ is a ladder with a spur or $((n-1) / n)$-external 2-cell at each end.

Observe that since $P$ and $J$ are locally injective, the only possible spurs are at their endpoints. Thus, if there are three spurs and/or $((n-2) / n)$-external 2 -cells then at least one of the 2-cells must lie along $P$ in the sense that it is a 2-cell $R$ with a path $Q$ in $\partial R$ such that $Q$ is a subpath of $P$ and $|Q|>\left|W^{n-2}\right|$. This is because at most two of the spurs and/or external 2-cells lie at the endpoints of $P$ and $J$, and since 
$((n-2) / n) \geq 1 / 2$, no such spur or 2-cell can lie along $J$ because it would contradict that $J$ is a geodesic. If $D$ is a ladder then either $|P| \leq K|J|$ or for some 2-cell $R$ at the end of $D$ there is a path $Q$ in $\partial R$ such that $Q$ is a subpath of $P$ and $|Q|>\left|W^{n-1}\right|$.

For a 1-cell $e$ of $X$ that occurs in the attaching map of $W^{n}$, we weight the sides at $e$ by $(1 / \#(e))$ where \#(e) is the number of times that $W$ passes through $e$. It follows that Perimeter $(e)$ is equal to $n$ or 0 according as to whether or not $e$ appears in the attaching map $W^{n}$ of the unique 2-cell $R$ of $X$. Let $S$ be the path such that $Q S$ is a cyclic conjugate of $W^{ \pm n}$ and observe that $|S|<2|W|$ and therefore Perimeter $(S)<2|W| n$. Next observe that Weight $(R) \geq 2 n$ because Weight $(R)$ is equal to $n$ times the number of distinct letters occurring in $W$ which we assumed to be at least 2 . Finally, since by hypothesis $n \geq|W|$, we combine these inequalities to obtain

$$
\text { Perimeter }(S)<2|W| n \leq 2 n n \leq n \text { Weight }(R)
$$

and we are done.

\section{Freiheitssatz and asphericity}

In this section we provide tower proofs of the Freiheitssatz and asphericity theorems for staggered 2-complexes. We refer the reader to [6, III.9.5] for a combinatorial proof of the Freiheitssatz for staggered presentations. Our proof follows Howie's approach in [3] very closely.

THEOREM 6.1. Let $X$ be a staggered 2-complex. Let $Z \subset X$ be a connected subcomplex with the property that

(1) if $C$ is a 2-cell of $X$ with $\partial C \subset Z$ then $C \subset Z$, and

(2) the ordered 1-cells of $X$ contained in $Z$ form an interval,

then $\pi_{1} Z \rightarrow \pi_{1} X$ is injective.

Proof. Consider a reduced disc diagram $\phi: D \rightarrow X$ for a closed path $P \rightarrow Z$. We will show that $D$ maps to $Z$ and thus $P$ is null-homotopic in $Z$.

If every ordered 1-cell in $\phi(D)$ is contained in $Z$, then by property 1, every 2-cell in $D$ would map to $Z$. Arguing by contradiction, suppose that some ordered 1-cell of $\phi(D)$ is not contained in $Z$. By reversing the ordering of the 1 -cells if necessary, we assume without loss of generality that $\phi(D)$ contains a greater 1-cell than $Z$ does. Now consider a maximal tower lift

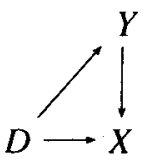


and observe that the top 1-cell $e$ of $Y$ does not map to $Z$. But $\partial D$ maps to $Z$ because $P$ factors through $\partial D$ and $P$ maps to $Z$. So no preimage of $e$ can lie in $\partial D$. It follows that since $e$ is in the image of $D \rightarrow Y, e$ must lie on the boundary of some 2-cell in $Y$. Consequently $e$ is $\max \alpha$ where $\alpha$ is the top 2-cell of $Y$. But this contradicts Lemma 4.1 which asserts that each preimage of $e$ lies in $\partial D$.

The following special case of the above result is a direct generalization of the classical Freiheitssatz for one-relator groups.

COROLlaRY 6.2. Let $X$ be a staggered 2-complex, and let $Y \subset X$ consist of the subgraph of unordered 1-cells, then for any choice of basepoints, $\pi_{1} Y \rightarrow \pi_{1} X$ is injective.

DEFINITION 6.3. The 2-complex $X$ is said to be combinatorially reducible provided that any spherical diagram $S \rightarrow X$ has a cancelable pair of 2-cells.

We refer the reader to Bogley and Pride [1, V.2.1] for a detailed account of the various asphericity notions that appear in the literature. Their account is not given in terms of diagrams, but rather in the equivalent language of pictures. Briefly, we note that if $X$ is combinatorially reducible and if no 2-cell of $X$ is attached by a proper power, and $X$ has no pair of 2-cells with the same attaching map, then $X$ is aspherical in the traditional sense that the universal cover $\tilde{X}$ is contractible.

The asphericity of the standard 2-complexes of cyclically reduced one-relator presentations where the relator is not a proper power was first determined by Lyndon in [5]. We also refer the reader to [6, III.9.7] for a combinatorial proof.

THEOREM 6.4. Every staggered 2-complex is combinatorially reducible.

ProOF. Let $S \rightarrow X$ be a reduced spherical diagram. Let $S \rightarrow Y$ be a maximal tower lift of $S \rightarrow X$. By Lemma 3.1, $S \rightarrow Y$ is reduced. To complete the proof, we apply Lemma 4.1 to see that there is a 1 -cell on $\partial S$ which is ridiculous.

\section{References}

[1] W. A. Bogley and S. J. Pride, 'Calculating generators of $\Pi_{2}$ ', in: Two-dimensional homotopy and combinatorial group theory (eds. C. Hog-Angeloni. W. Metzler and A. J. Sieradski) (Cambridge Univ. Press, Cambridge, 1993) pp. 157-188.

[2] J. Howie, 'On pairs of 2-complexes and systems of equations over groups', J. Reine Angew. Math. 324 (1981), 165-174.

[3] — 'How to generalize one-relator group theory'. in: Combinatorial group theory and topology (eds. S. M. Gersten and J. R. Stallings) (Princeton Univ. Press, Princeton, N.J., 1987) pp. 53-78. 
[4] J. Howie and S. J. Pride, 'A spelling theorem for staggered generalized 2-complexes, with applications', Invent. Math. 76 (1984), 55-74.

[5] R. C. Lyndon, 'Cohomology theory of groups with a single defining relation', Ann. of Math. (2) $52(1950), 650-665$.

[6] R. C. Lyndon and P. E. Schupp, Combinatorial group theory, Ergebnisse der Mathematik und ihrer Grenzgebiete, Band 89 (Springer, Berlin, 1977).

[7] W. Magnus, 'Über diskontinuierliche Gruppen mit einer definierenden Relation (Der Freiheitssatz)', J. Reine Angew. Math. 163 (1930), 141-165.

[8] — , 'Das Identitätsproblem für Gruppen mit einer definierenden Relation', Math. Ann. 106 (1932), 295-307.

[9] J. P. McCammond and D. T. Wise, 'Coherence, local quasiconvexity and the perimeter of 2 complexes', preprint, 1999.

[10] _ 'Fans and ladders in small cancellation theory', Proc. London Math. Soc., to appear.

[11] B. B. Newman, 'Some results on one-relator groups', Bull. Amer. Math. Soc. 74 (1968), 568-571.

[12] C. D. Papakyriakopoulos, 'On Dehn's lemma and the asphericity of knots', Ann. of Math. (2) 66 (1957), 1-26.

[13] H. Short, 'Quasiconvexity and a theorem of Howson's', in: Group theory from a geometrical viewpoint (Trieste, 1990) (eds. É. Ghys, A. Haefliger and A. Verjovsky) (World Sci. Publishing, River Edge, NJ, 1991) pp. 168-176.

Department of Mathematics

Cornell University

Ithaca, NY 14853

USA

e-mail: chruska@math.cornell.edu
Department of Mathematics and Statistics

McGill University

Montreal, Quebec H3A ZK6

Canada

e-mail: wise@math.mcgill.ca 